Meta

Journal des traducteurs

Translators' Journal

\title{
Bibliographie - Écologie des eaux continentales - Classification par domaine
}

\section{Philippe Thoiron et André Clas}

Volume 36, numéro 4, décembre 1991

URI : https://id.erudit.org/iderudit/004481ar

DOI : https://doi.org/10.7202/004481ar

Aller au sommaire du numéro

Éditeur(s)

Les Presses de l'Université de Montréal

ISSN

0026-0452 (imprimé)

1492-1421 (numérique)

Découvrir la revue

Citer ce document

Thoiron, P. \& Clas, A. (1991). Bibliographie - Écologie des eaux continentales - Classification par domaine. Meta, 36(4), 653-662.

https://doi.org/10.7202/004481ar d'utilisation que vous pouvez consulter en ligne. 


\section{Bibliographie - Écologie des eaux continentales - Classification par domaines}

Cette bibliographie a été élaborée dans le cadre du programme de recherche intégrée France - Québec (Responsable: André Clas et Philippe Thoiron). Elle a été compilée, sous la supervision de Janine Gibert (Université Claude Bernard, Lyon), avec l'assistance de Laurence Batista, Véronique Viretto, Jean-Luc Maaded (Université Lumière, Lyon), Jérôme Guillouët, (Université Claude Bernard, Lyon), et Louis-Paul Rioux, Anne-Marie Nadeau, Carole Poisson, Marie-Hélène Cartier, Chantal Labrie (Université de Montréal).

- INTRODUCTION AU DOMAINE (LIMNOLOGIE/ÉCOLOGIE):

BARNES, R.S.K., MANN, K.H. (1980): Fundamental of Aquatic Ecosystems, London, Blackwell Scientific Publications, $299 \mathrm{p}$. 
BERNARDIS, M.A. (1990): Le Grand livre de l'eau, Paris, la Manufacture et la Cité des sciences et de l'industrie, $410 \mathrm{p}$.

CHAMPIAT, D., LARPENT, J.P. (1988): Biologie des eaux, Méthodes et techniques, Paris, Masson, 371 p.

DUVAL, Clément (1971): L'Eau, Que sais-je? n 884, Paris, PUF, 128 p.

KORMONDY, Edward J. (1984): Concepts of Ecology, New Jersey, Prentice-Hall, 3rd ed.

ODUM, Eugène P. (1976) : Écologie, traduction de R. Bergeron, Paris, Doin, Montréal, HRW

PERES, Jean-Marie (1972): La Vie dans les mers, Que sais-je ? n 72, Paris, PUF, $126 \mathrm{p}$.

PYTKOWICZ, Ricardo M. (1983): Equilibria, Nonequilibria, and Natural Waters, Vol. 1, New York, John Wiley \& Sons, 331 p.

ROCHEFORT, Michel (1969) : Les Fleuves, Que sais-je ? $\mathrm{n}^{\circ}$ 1077, Paris, PUF, 128 p.

WELCH, Paul S. (1952): Limnology, New York, McGraw-hill, 2nd ed., 538 p.

\section{AGRICULTURE :}

DURAND, Jacques H. (1983): Les Sols irrigables : étude pédologique, Paris, PUF, 339 p.

HENIN, S., GRAS, R., MONNIER, G. (1969): Le Profil cultural, Paris, Masson, 332 p.

INTERNATIONAL INSTITUTE FOR LAND RECLAMATION AND IMPROVEMENT/ILRI (1979): Drainage Principles and Applications, Vol. 1, Introductory Subjects, Wageningen, The Netherlands, International Institute for Land Reclamation and Improvement / ILRI, 2nd ed., (1st ed. 1972), $470 \mathrm{p}$.

INTERNATIONAL INSTITUTE FOR LAND RECLAMATION AND IMPROVEMENT/ILRI (1979): Drainage Principles and Applications, Vol. 2. Wageningen, The Netherlands, International Institute for Land Reclamation and Improvement / ILRI, 2nd ed., $470 \mathrm{p}$.

INTERNATIONAL INSTITUTE FOR LAND RECLAMATION AND IMPROVEMENT/ILRI (1980): Drainage Principles and Applications, Vol. 4, Design and Management of Drainage Systems, publication 16 vol., iv. Wageningen, The Netherlands, International Institute for Land Reclamation and Improvement / ILRI, 2nd ed., (1st ed. 1974), $470 \mathrm{p}$.

PENKA, M., VYSKOT, M. (1985): Developments in Agricultural and Managed-forest Ecology 15A: Floodplain forest ecosystem: 1. Before water management measures, Amsterdam, Elsevier, $466 \mathrm{p}$.

POIREE, M., OLLIER, CH. (1978): Assainissement agricole - Drainage par tuyaux ou fossés, aménagement des cours d' eau et émissaires, Bibliothèque de l'Institut de topométrie, Paris, Eyrolles, $519 \mathrm{p}$.

SMEDEMA, Lambert K., RYCROFT, David W. (1983): Land Drainage, Planning and Design of Agricultural Drainage Systems, Ithaca, New York, Cornwell University Press, 376 p.

\section{ALGUES ET PLANTES AQUATIQUES :}

LIETH, Helmut, WHITTAKER, Robert H. (Eds.) (1975): Primary Productivity of the Biosphere, Berlin, Springer, $339 \mathrm{p}$.

SHUBERT, L. Elliott (Ed.) (1984): Algae as Ecological Indicators, London, Academic Press, XII + 434 p.

\section{- AMÉNAGEMENT DES COURS D'EAU/HYDROÉLECTRICITÉ:}

BEDAUX, Robert (1978): Terrassement dans les terrains aquifères, Paris, Technique et documentation, $130 \mathrm{p}$.

BROOKES, A. (1988): Channelized Rivers Perspectives for environmental Management, Chichester, John Wiley \& Sons, $326 \mathrm{p}$.

DAWSON, John A., DOORNKAMP, John C. (1973): Evaluating the Human Environment - Essays in Applied Geography, New York, St-Martin's Press, $288 \mathrm{p}$.

HARRIS, Robert (1969): Canals and their Architecture, London, Hugh Evelyn, 223 p.

JAMES, L. Douglas, LEE, Robert R. (1971): Economics of Water Resources Planning, McGraw-Hill series in Water Resources and Environmental Engineering, New York, McGraw-Hill, 615 p.

PASKOFF, R. (1985): Les Littoraux: impact des aménagements sur leur évolution, Coll. Géo., Paris, Masson, $184 \mathrm{p}$.

LELIAVSKY, S. (1961): Précis d' hydraulique fluviale, Paris, Dunod, $256 \mathrm{p}$.

LORIFERNE, M. (dir.) (1975): Approche écologique des retenues d'eau pluviale, $88 \mathrm{p}$.

MARY, Marcel (1965): Les Barrages, Que sais-je? n ${ }^{\circ} 1183$, Paris, PUF, 127 p.

MARY, Marcel, JANOD, Albert (1967): La Houille blanche, Que sais-je? n ${ }^{\circ}$ 540, Paris, PUF, $128 \mathrm{p}$.

SMITH, Robert Leo (1976): The Ecology of Man: An Ecosystem Approach, New York, Hagerstown, San Francisco, London, Harper and Row, 2nd ed., (1st ed. 1972), 400 p.

THOMAS, Henry H. (1976): The Engineering of Large Dams, part I, London, New York, Sydney, Toronto, John Wiley \& Sons, $376 \mathrm{p}$ 
BACTÉRIES :

FLETCHER, M., GRAY, T.R.G., JONES, J.G. (Eds.) (1987): Ecology of Microbial Communities, Cambridge, Cambridge University Press.

CLIMATOLOGIE :

ARLERY, R., GUILMET, B., GRISOLLET, H. (1973): Climatologie, méthodes et pratiques, Paris, GauthiersVillars \& Cie.

ATKINSON, Bruce W. (1970): Observation, analyse, précision et modification du temps, Paris, Larousse.

BARRETT, E.C. (1974): Climatology from Satellites, London, Methuen Company Ltd.

BARRY, R.G., CHORLEY, R.J. (1968): Atmosphere, Weather and Climate, London, Methuen Company Ltd.

BATTAN, Louis J. (1985): Weather, New Jersey, Englewood Cliffs, Prentice-Hall, 2nd ed.

CHANG, Jen-Hu (1968): Climate and Agriculture: an Ecological Survey, Chicago, Aldine Publishing Company, $304 \mathrm{p}$.

CRITCHFIELD, Howard J. (1966): General Climatology, New Jersey, Englewood Cliffs, Prentice-Hall.

CROWE, P.R. (1971): Concepts in Climatology, London, Longman Group Limited, 589 p.

DE LONG, George C., KOEPPE, Clarence E. (1958): Weather and Climate, New York, McGraw-Hill.

DIMACOPOULOS, G., DURAND, R. (1969): Éléments de météorologie agricole, Paris, J.B. Baillière \& Fils.

DUFOUR, L. (1970): Les Particules de glace atmosphériques, Institut royal météorologique de Belgique.

DURHAM, Jack L. (1984): Fogs and Rain, (Chemistry of Particles), Toronto, Ontario, Butterworth Publishers.

DYSON, James L. (1963): The World of Ice, New York, Alfred A. Knopf.

ESCOURROU, Gisèle (1978): Climatologie pratique, Paris, Masson.

ESTIENNE, Pierre, GODARD, Alain (1970): Climatologie, Paris, Armand Colin.

FLETCHER, N.H. (1970): The Chemical Physics of Ice, London, Cambridge University Press.

FOURNIER, Frédéric (1960): Climat et érosion: la relation entre l'érosion du sol par l'eau et les précipitations atmosphériques, Paris, PUF, 198 p.

GOODY, R.M., WALKER, J.C.C. (1972): Atmospheres, New Jersey, Prentice-Hall.

HUFTY, A. (1976): Introduction à la climatologie, Paris, PUF.

KNOWLES, W.E. (1965): A History of the Theories of Rain, London, Oldburne Book Company Ltd.

LIMBREY, Susan (1975): Soil Science and Archaelogy, London, Academic Press, 384 p.

LLIBOUTRY, L. (1964): Traité en glaciologie, t. I. Paris, Masson.

LOCKWOOD, J.G. (1979): Causes of Climate, New York, A Halsted Wiley Press Book, John Wiley \& Sons, $260 \mathrm{p}$.

MASON, B.J. (1975): Clouds, Rains and Rainmaking, Cambridge, England, Cambridge University Press.

MONEY, D.C. (1965): Climate, Soils and Vegetation, London, University Tutorial Press Ltd., 272 p.

NEIBURGER, M. (1973): Understanding our Atmospheric Environment, San Francisco, W.H. Freeman and Company, $293 p$

NIVEN, C.D. (1963): The Magic Surface, New York, Pageant Press Inc.

OLIVER, John E. (1973): Climate and Man's Environment, (An Introduction to Applied Climatology), Toronto, Canada, John Wiley \& Sons.

OLIVIER, J.E., HIDORE, J.J. (1984): Climatology, an Introduction, Toronto, Canada, Charles E. Merrill Publishing Company, A Bell and Howell Company, $381 \mathrm{p}$.

PEGUY, CH.P. (1961): Précis de climatologie, Paris, Masson, 347 p.

SCHOUMSKY, P.A. (1957): Principes de glaciologie structurale, Paris, Centre national de la recherche scientifique.

SHUMSKII, P.A. (1964): Principles of Structural Glaciology, New York, Dover Publications Inc.

VIAULT, André (1973): La Météorologie, Que sais-je ? n ${ }^{\circ} 89$, Paris, PUF, 2 éd. mise à jour.

VIERS, G. (1971): Éléments de climatologie, Paris, Fernand Nathan, éd. revue et corrigée.

WEAVER, John C. (Consulting Ed.) (1954): An Introduction to Climate, New York, McGraw-Hill.

\section{I. ÉCOLOGIE:}

ALLEE, W.C., SCHMDT, K.P. (1951): Ecological Animal Geography, London, Chapman \& H \& Hall Ltd, $715 \mathrm{p}$.

BARBAULT, R., BLANDIN, P., MEYER, J.A. (1980): Recherches d'écologie théorique, les stratégies adaptatives, Actes du Colloque d'écologie théorique organisé à l'École Normale supérieure de Paris, les 18 , 19, et 20 mai 1978, Paris, Maloine, 298 p.

BENTON, Allen H., WERNER, Jr., WILLIAM, E. (1966): Field Biology and Ecology, London, McGraw-Hill, $499 \mathrm{p}$. 
CLEMENTS, F.E., SHELFORD, U.E. (1939): Bio-Ecology, New York, John Wiley \& Sons, 425 p.

CRAIG, J.F., KEMPER, J.B. (1987): Regulated Streams, Advances in Ecology, London, Plenum Press, 431 p.

DREUX, D. (1974): Précis d'écologie, Paris, PUF, (3e éd. 1986).

DUMONT, R. (1976): L'Homme et son Environnement, de la démographie à l'écologie, Paris, Les encyclopédies du savoir moderne, Retz, $511 \mathrm{p}$.

EWEL, K.C., ODUM, H.T. (1986): Cypress Swamps, Gainesville, University of Florida Press, Center for Wetlands, $472 \mathrm{p}$.

FARB, Peter (1965): L'Écologie, Coll. Life, traduction de Paul Dunand, Time Life, 191 p.

FAURIEE, C., FERRA, C., MEDORI, P. (1978) : Écologie, Paris, J.B. Baillière, 147 p.

GODRON, M. (1989): Écologie de la végétation terrestre, Paris, Masson, $196 \mathrm{p}$.

KEENLEYSIDE, M.H.A. (1979): Diversity and Adaptations in Fish Behaviour, Berlin, Heidelberg, Springer, $208 \mathrm{p}$.

KENDEIGH, S.C. (1961): Animal Ecology, Illinois, Prentice-Hall, 468 p.

KREBS, C.I. (1972): Ecology, The Experimental Analysis of Distribution and Abundance, London, 694 p.

LAUREC, A., LECRUEN, J.C., (s.d.) Dynamique des populations marines exploitées (TI concepts et modèles), rapp. scient. et techn., $\mathrm{n}^{\circ} 45$, Brest, publication du CNEXO, CNEXO/CO13 Doc. $118 \mathrm{p}$.

LEBRETON, P. (1978) : Éco-logique, initiation aux disciplines de l' environnement, Paris, Inter-Éditions, $239 \mathrm{p}$.

LEGAY, J.M., TOMASSONE, R. (1978) : Biométrie et écologie, Paris, Société française de biométrie, 387 p.

McDOWALL, R.M. (1988): Diadromy in Fishes (Migrations between Freshwater and Marine Environments), London, Croom Helm II, 308 p.

McKEOWN, B.A. (1984): Fish Migration, Beckenham, Croom Helm Ltd, 224 p.

MAY, R.M. (1981): Theoretical Ecology, Principles and Applications, London, Blackwell Scientific Publications, 2nd ed., $489 \mathrm{p}$.

MEDBAG, FRA, Toulouse, CACG, FRA, Tarbes (1987): Inventaire écologique de la Garonne (synthèse), Haute Garonne, Tarn et Garonne, Lot et Garonne, Gironde, 4 volumes N.p., C. Toulouse, Minist. Environ. Bassin Adour-Garonne; Tarbes, Comp. Amen. C. Gasc.

MOLINIER, R., VIGNES, P. (1971): Écologie et biocénotique, les êtres vivants, leurs milieux, leurs communautés, l'environnement, Neuchâtel, Suisse, Delachaux et Niestlé, $457 \mathrm{p}$.

ODUM, E.P. (1976): Écologie, traduction de Raymond Bergeren, Montréal, MRW, ODECO, 281 p.

POLUMIN, N. (1986): Ecosystem Theory and Application, New York, John Wiley \& Sons, 445 p.

RAMADE, F. (1989): Élément d'écologie fondamentale, Paris, McGraw-Hill, 397 p.

RAST, W., HOLLAND, M., RYDING, S.O. (1989): Eutrophication Management Framework for the Policymaker, Paris, Unesco, 83 p.

RYDING, S.O., RAST, W. (1989): Man and the Biosphere Series: the Control of Eutrophication of Lakes and Reservoirs, Vol. I, Paris, Unesco and The Parthenon Publishing Group, 314 p.

SACCHI, C.F., TESTARD, P. (1971) : Écologie animale, organismes et milieu, Coll. Biologie, Paris, Doin, 479 p.

p.
WELLS, S.M., PYLE, R.H., COLLINS, N.M. (1984): The IVCN Invertebrate Red Data Book, Cambridge, Cambridge University Press, $630 \mathrm{p}$.

WILSON, Edward O. (1975): Sociobiology, the New Synthesis, Boston, Belkrap-Harvard, 697 p.

\section{EAUX SOUTERRAINES :}

AMERICAN WATER WORKS ASSOCIATION (1973): Groundwater, New York,

BITTON, G., GERBA, C.P. (1984): Groundwater Pollution Microbiology, New York, John Wiley \& Sons, 377 p.

BOONSTRA, J. (1981): Numerical Modelling of Groundwater Bassins, Netherlands, International Institute for Land Reclamation and Improvement.

BOWEN, R. (1986): Groundwater, London, Elsevier, 2nd ed., 427 p

CASTANY, G. (1967): Traité pratique des eaux souterraines, Paris, Dunod, 661 p.

DOMENICO, P.A. (1972): Concepts and Models in Groundwater Hydrology, New York, McGraw-Hill.

HARR, M.E. (1962): Groundwater and Seepage, New York, McGraw-Hill.

HUISMAN, L. (1972): Groundwater Recovery, New york, Winchester Press.

MARINO, M.A. (1982): Seepage and Groundwater, Netherlands, Elsevier Scientific Publishing Company.

MATTHES, G. (1982): The Properties of Groundwater, New York, John Wiley \& Sons, $406 \mathrm{p}$.

MEINSER, O.E. (1923): Outline of Groundwater Hydrology, Washington, Washington Government Printing Office.

NATIONS UNIES (1977): Les Eaux souterraines dans l' hémisphère occidental, New York, Nations Unies.

PLOTNIKOV, N.A. (1962): Ressources en eaux souterraines: classification et méthodes d'évaluation, Paris, Gauthier-Villars, $194 \mathrm{p}$.

RALPH, C. (1968): Groundwater hydrology, New York, John Wiley \& Sons. 
RAUDKIVI, A.J. (1976): Analysis of Groundwater Flow, London, Edward Arnold Ltd. SCHOELLER, H. (1974): Les Eaux souterraines, Paris, Masson.

TROMBE, F. (1950): Les Eaux souterraines, Que sais-je? $\mathbf{n}^{\circ} 455$, Paris, PUF, 128 p.

TROMBE, F. (1969): Les Eaux souterraines, Paris, PUF.

VERRVIJT, A. (1970): Theory of Groundwater Flow, New York, Gordon and Breach Science Publisher Inc.

WALTON, W.C. (1970): Groundwater Resource Evaluation, New York, McGraw-Hill.

- GÉOLOGIE/GÉOMORPHOLOGIE / SÉDIMENTOLOGIE :

BABAULT, R. (1983): Abrégé de géologie générale, Paris, Masson, $224 \mathrm{p}$ BELLAIR, P., POMEROL, C. (1971): Eléments de géologie, Paris, Armand Colin, 3 e ed., 526 p. BOILLOT, G. (1990): Géologie des marges continentales, Paris, Masson, 135 p.

BOULIN, J. (1977): Méthodes de la stratigraphie et géologie historique, Paris, Masson, $226 \mathrm{p}$.

BRAVARD, J.P. (1987): Le Rhône, du Léman à Lyon, Coll. L'homme et la nature, Lyon, La manufacture, 451 p.

CAMPY, M., MACAIRE, J.J. (1989) : Géologie des formations superficielles, Paris, Masson, 433 p.

CHAMLEY, H. (1988): Les Milieux de sédimentation, Paris, 123 p.

CHAMLEY, H. (1987): Sédimentologie, Paris, Dunod Université, Géosciences, Bordas, 175 p.

COQUE, R. (1977): Géomorphologie, Paris, Université Paris, Armand Colin, 430 p.

DEMANGEOT, J. (1990) : Les Milieux «naturels» du globe, Coll. Géographie, Paris, Masson, $3 e$ éd., 276 p.

DERRUAU, M. (1979): Les Formes du relief terrestre, Paris, Masson, 120 p.

DERRUAU, M. (1986): Les Formes du relief terrestre, Paris, Masson, 4 e éd., 119 p.

DERRUAU, M. (1965): Précis de géomorphologie, Paris, Masson, $415 \mathrm{p}$.

DERRUAU, M. (1988): Précis de géomorphologie, Paris, Masson, 7 éd., 533 p.

DESITTER, L.U. (1964): Structural Geology, London, GB, McGraw-Hill, 551 p.

KNIGHTON (1984): Fluvial Forms and Processes, Arnold, 218 p.

LEBRETON, J.C. (1974): Dynamique fluviale, Paris, Eyrolles.

MORISAWA, M. (1981): Fluvial Geomorphology, George Allen \& Cie, 314 p.

MORISAWA, M. (1985): Rivers, London, Longman, 222 p.

PETTS, G.E. (1983): Impounded Rivers, Perspectives for Ecological Management, $326 \mathrm{p}$.

PETTS, G.E., FOSTER, I., (1985): Rivers and Landscape, Arnold, 273 p.

REINECK, M.E., SINGH, I.B. (1980): Depositional Sedimentary Environments - Fluvial Environment,

New York, Berlin, Springer-Verlag, 549 p., Chap. 2, pp. 257-31.

HODES, D.D., WILLIAMS, G.I. (1979): Adjustments of the Fluvial System, 372 p.

RICHARDS, K. (1982): Rivers, Form and Process in Alluvial Channels, New York, Methuen \& Co., 360 p.

RIES, H., WATSON, T.L. (1953): Engineering Geology, London, John Wiley \& Sons Inc., 3rd ed., 750 p.

SCHUM, S.A. (1977): The Fluvial System, London, John Wiley \& Sons, 338 p.

SCHUM, S.A., HARVEY, M.D., WATSON, C.C. (1984): Incised Channels, Morphology, Dynamics and Control, Water Ressources Publications, $200 \mathrm{p}$.

SCHWARTZ, G..M., THIEL, G.A. (1963): Minnesota's Rocks and Waters, a Geological Story, Minneapolis, USA, The University of Minnesota Press, $366 \mathrm{p}$.

SIFFRE, M. (1981): Grottes, gouffres et abîmes, Paris, Hachette réalités, Les quatre éléments, 157 p.

STEILA, Donald (1976): The Geography of Soils, New jersey, Prentice-Hall, 222 p.

TRICART, J. (1978): Géomorphologie applicable, Coll. de géographie applicable, Paris, Masson, 240 p.

TRICART, J. (1981): Géomorphologie climatique, Paris, SEDES, 313 p.

TRICART, J. (1977): Géomorphologie dynamique générale, Paris, SEDES, 345 p.

TRICART, J. (1968): Géomorphologie structurale, Paris, SEDES, 322 p.

TRICART, J. (1977): Précis de géomorphologie, t. I et t. II, Paris, CDU et SEDES, 345 p. + 322 p.

TRICART, J. (1965): Principes et méthodes de la géomorphologie, Paris, Masson, 484 p.

TRICART, J., CAILLEUX, A. (1962): Le Modele glaciaire et nival, Paris, SEDES, 508 p.

TRICART, J., CAILLEUX, A. (1967): Le Modèle des régions périglaciaires, Paris, SEDES, $512 \mathrm{p}$.

TRICART, J., KILLIAN, J. (1979): L'Éco-géographie et l'Aménagement du milieu naturel, Paris, Maspero, $320 \mathrm{p}$.

VIERS, G. (1967) : Éléments de géomorphologie, Paris, Nathan, 207 p.

ZACHAR, Dusan (1982): Soil Erosion, Developments in Soil Science, New York, Elsevier Scientific Publishing Company, $547 \mathrm{p}$

HYDROLOGIE :

BERNER, E.K. (1987): The Global Water Cycle, New Jersey, Prentice-Hall.

BRUTSAERT, W. (1982): Evaporation into the Atmosphere: Theory, History and Applications, Boston, D. Reidel Publishing Company. 
CASTANY, G. (1982): Principes et méthodes de l' hydrologie, Paris, Dunod Université, Bordas, 238 p.

CHOW, Ven Te (1964): Handbook of Applied Hydrology, A Compendium of Water-Resources Technology, New York, McGraw-Hill.

FETLER, C.W. (1980): Applied Hydrology, Bell and Howell Company.

FRECAULT, R. (1964) : Eléments d' hydrologie continentale, Paris, Centre de documentation universitaire.

GRAY, D.M. (1970): Handbook on the Principles of Hydrology, Ottawa, National Research Council of Canada.

GUYOT, Charles (1974): L'Hydrologie, Que sais-je ? n ${ }^{\circ} 884$, Paris, PUF, 129 p.

HILLEL, Danicl (1971): Soil and Water, Physiological Ecology, A Series of Monographs, Texts and Treatises, New York, Academic Press, 288 p.

LEOPOLD, B. (1966): Water, New York, Time Inc.

LINSLEY (1975): Hydrology for Engineers, New York, McGraw-Hill.

MILLER, D.H. (1977): Water at the Surface of the Earth, New York, Academic Press.

MORETTE, A. (1964): Précis d' hydrologie, Paris, Masson.

NATIONAL RESEARCH COUNCIL ASSOCIATE COMMITTEE ON GEODESY AND GEOPHYSICS (1973): Fluvial Processes and Sedimentation - Proceedings of Hydrology Symposium held at the University of Alberta, Edmonton, May 8 and 9, 1973, Ottawa, Canada, National Research Council Associate Committee on Geodesy and Geophysics, Subcommittee on Hydrology, $759 \mathrm{p}$.

RAPHAEL, G. (1972): Modern Hydrology, New York.

ROGER, J.M. (1965): Geohydrology, New York, John Wiley \& Sons.

SMITH, D.I., STOPP, P. (1978): The River Basin. An Introduction to the Study of Hydrology, London, Cambridge University Press, F.C. Evans and M.A. Morgan eds., 120 p.

STUMM, W. (1985): Chemical Processes in Lakes, New York, John Wiley \& Sons, 435 p.

THURMAN, E.M. (1985): Developments in Biogeochemistry: Organic Geochemistry of Natural Waters, Dordrecht, Martinus Nijhoff, Dr. W. Junk Publishers, 497 p.

UNESCO (1985): Technical Papers in Hydrology 27: Teaching Aids in Hydrology, A Contribution to the International Hydrological Programme, Paris, Unesco, $76 \mathrm{p}$.

WISLER, C.O., BRATER, E.F. (1959): Hydrology, New York, John Wiley \& Sons Inc., London, Chapman and Hall, Ltd, 2nd ed., 408 p.

- ICHTYOLOGIE:

BAUCHOT, R. et M.L. (1964): Les Poissons, Que sais-je ? n 642, Paris, PUF, 126 p.

KISTER, René (Éd.): Le Grand livre de la mer et des poissons, Monaco, Kister René éd.

LAGLER, K.F., BARDACH, J.E., MILLER, R.R. (1967): Ichthyology, New York, London, Sidney, John Wiley \& Sons Inc., $545 \mathrm{p}$.

LEGENDRE, Vianney, BERGERON, Jacques F., Nomenclature de stades du saumon atlantique, Salmo salar Linné 1758, Montréal, Canada, Service de l'aménagement et de l'exploitation de la faune.

LEIM, A.M., SCOTT, W.B. (1966): Fishes of the Atlantic Coast of Canada, Bull. $\mathrm{n}^{\circ}$ 155, Ottawa, Canada, Fisheries Research Board of Canada, $485 \mathrm{p}$.

MOYLES, P.B., CECK, J.J. (1988): Fishes: an Introduction to Ichtyology, New Jersey, Englewood-Cliffs, Prenctice-Hall, 2nd ed.

PINCHER, C. (1948): A Study of Fish, New york, Duell, Sloane and Pearce, 343 p.

- INONDATIONS / CRUES/ÉTIAGES:

HANDMER, John (Ed.) (c1987): Flood Hazard Management, British and International Perspectives, Norwich, GB, Geo Books, 297 p.

HOYT, W.G., LANGBEIN, W.B. (1955): Floods, Princeton, N.J., Princeton University Press, 469 p.

HUMBERT, J., ClOOTS, A.R., MAIRE, G. (1987): Actes du Colloque: crues et inondations (Génèse, méthodes d'étude, impacts et prévention), 16-18 octobre 1986, Strasbourg, Strasbourg, Université L. Pasteur, Centre d'étude et de recherche écogéographiques, $363 \mathrm{p}$.

LARRAS, Jean (1975) : Défense contre les crues, Paris, Eyrolles, $135 \mathrm{p}$.

LARRAS, Jean (1972): Prévision et prédétermination des étiages et des crues, Coll. du Bureau central d'études pour les équipements d'outre-mer (B.C.E.O.M.), Paris, Eyrolles, 159 p.

\section{MÉTHODES DE MESURE :}

RICKER, W.E. (1975): Computation and Interpretation of Biological Statistics of Fish Populations, Bull. Fish. Res. Board, Can. Bull. 191, 382 p.

ROCHICCIOLO-DELTCHEFF, Cl. (1970): Le pH et sa mesure, Que sais-je ? n 1248, Paris, PUF, 126 p. 


\section{- OCÉANOGRAPHIE:}

DIETRICH, G. (1943): General Oceanography, An Introduction, New York, John Wiley \& Sons, 588 p. PEREZ, J.M. (1961): Océanographie biographique et biologie marine, Vol. I, Paris, PUF, 541 p. PEREZ, J.M. (1961): Océanographie biographique et biologie marine, Vol. II, Paris, PUF, 514 p.

STANDRING, G. (1978): Les Mers et les Océans, Coll. Terre vivante, vol. 5, 9e partie, traduction de G. et R. Baschet, Paris, Chantereine, 143 p.

- PÊCHES / PISCICULTURE :

ANDREEV, N.N. (1966): Handbook of Fishing Gear and its Rigging, traduit du russe, Jérusalem, Israël Program for Scientific Translations, $454 \mathrm{p}$.

BOYER, Albert (1967): Les Pêches maritimes, Que sais-je ? nº 199, Paris, PUF, 126 p.

BROWNING, R.J. (1980): Fisheries of the North Pacific History, Species, Gear and Processes, Alaska Northwest Publishing Company, 423 p.

EVERHART, Harry W., YOUNGS, William D. (1981): Principles of Fishery Science, London, Cornwall University Press, 2nd ed., (1st ed. 1975), 349 p.

JAMET, J., LAGOIN, Y. et coll. (1974): Manuel des pêches maritimes tropicales, Paris, ministère de la Coopération, $447 \mathrm{p}$

JONES, R. (1979): Materials and Methods Used in Marking Experiment in Fishery Research, Rome, FAO Fish Tech. Pap. 190, $134 \mathrm{p}$

JOHNSON, D.C., NIELSON, L.A. (1983): Fisheries Techniques, Virginia, the American Fisheries Society, Southern Printing Company Inc., $468 \mathrm{p}$.

MIGDALSKI, Edward C. (1958): Angler's Guide to the Salt Water Game Fishes, New York, The Ronald Press Company.

MONGEAU, J.R. (1976): Méthodes de pêche expérimentale, en eau douce, à l'usage du biologiste et du technicien de la faune, Montréal, Canada, Service de l'aménagement de la faune, ministère du Tourisme, de la chasse et de la pêche, $83 \mathrm{p}$.

MOUSSETTE, M. (1979): La pêche sur le St-Laurent, Répertoire des méthodes et des engins de capture, Montréal, Canada, Boréal express, 212 p.

NEDELEC, C. (1982): Définition et classification des catégories d'engins de pêche, Rome, FAO, Doc. Tech Pêches (222), $51 \mathrm{p}$.

PITCHER, T.J., HART, P.J.B. (1982): Fisheries Ecology, AVI Publishing Company Nertport, 414 p.

ROUNSEFELL, G.A., EVERHART, W.H. (1953): Fishery Science: its Methods and Applications, New York, John Wiley \& Sons, London, Chapman and Hall Ltd, 444 p.

\section{PLANCTON/BENTHOS :}

BOUGIS, P. (1974): Écologie du plancton marin, le phytoplancton, t. I, Coll. d'écologie ${ }^{\circ} 2$, Paris, Masson. BOUGIS, P. (1974): Écologie du plancton marin, le zooplancton, t. II, Coll. d'écologie ${ }^{\circ} 3$, Paris, Masson.

EHRHARDT, J.P., SEGUIN, G. (1978): Le plancton, composition, écologie, pollution, Paris, Gauthier-Villars, Bordas, $210 \mathrm{p}$.

GAUTHIER, Benoît (1979): Présentation du phytobenthos Limnétique, mémoire de la Société limnéenne du Québec, Québec, $78 \mathrm{p}$.

McCAFFERTY, W.P. (1981): Aquatic Entomology. The Fishermen's and Ecologists' Illustrated Guide to Insects and their Relations, Boston, Science Books Intemational, $496 \mathrm{p}$.

NEWELL, G.E., NEWELL, R.C. (1963): Marine Plankton, London, Hutchinson Educational Ltd, 207 p.

\section{POLLUTION/EAUX POTABLES / TRAITEMENT DES EAUX:}

ASSOCIATION QUÉBÉCOISE DES TECHNIQUES DE L'EAU (1978): L'Eau, une industrie, congrès conjoint: AQTE FACE 14-17 mai 1978, 30-7 p.

GORE, J.A. (1985): The Restoration of Rivers and Streams. Theories and Experience, Boston, Butterworth Publishers, An Ann Arbor Science Book, 280 p.

HELLAWELL, J.M. (1989): Biological Indicators of Freshwater Pollution and Enviromnental Management, London, $546 \mathrm{p}$.

JAMES, G.V. (1971): Water Treatment - A Survey of current methods of purifying domestic supplies and of treating industrial effluents and domestic sewage, Cleveland, Ohio, CRC Press, 4th ed., (1st publ. 1940), $311 \mathrm{p}$. 
JOLÁNKAI, G., ROBERTS, G. (1985): Programme on Man and Biosphere: Project 5 workshop: Contamination of Subsurface Water Resources by Nitrate, 25/29 November 1985, Budapest, Hungary, Budapest, Vízügyi Dokumentációs Szolgáltató Leányvállalat nyomdája, 330 p.

OMS (1985): Directives de qualité pour l'eau de boisson, Vol. 1 Recommandations, Genève, OMS, 131 p.

OMS (1981): Eau potable et assainissement, 1981-1990 - Vers une meilleure santé - Contribution de l'OMS à la Décennie internationale de l'eau potable et de l'assainissement, Genève, OMS, $59 \mathrm{p}$.

OMS (1977): Surveillance de la qualité de l' eau de boisson, série de monographies $\mathrm{n}^{\circ} 63$, Genève, OMS, $143 \mathrm{p}$.

SAFE DRINKING WATER COMMITTEE (1980): Drinking Water and Health, Vol. 2, Board on Toxicology and Environmental Health Hazards, Assembly of Life Science, National Research Council, Washington D.C., National Academy Press, 393 p.

TURK, Amos, TURK, Jonathan, WITTES, Janet T. (1972): Ecology Pollution Environment, PhiladelphiaLondon-Toronto, W.B. Saunders Company, 217 p.

ULP, FRA, Strasbourg, XILLIOX, L., BELLER, C. (1987): Commission interministérielle d'étude de la nappe phréatique plaine d'Alsace: carte du risque de lessivage des nitrates dans les sols, $f, 8$, Neuf-Brisach, Strasbourg, Université L. Pasteur, Centre d'étude de recherche éco-géographiques, 23 p., Cht.

WAITE, Thomas D. (1984): Principles of Water Quality, Orlando, FLorida, Academic Press Inc., 289 p.

WILLIAMS, G.P., WOLMAN, M.G. (1984): Downstream Effects of Dams on Alluvial Rivers, Washington, Geological survey, $63 \mathrm{p}$

WORLD HEALTH ORGANIZATION (1981): Drinking Water and Sanitation, 1981-1990-A Way to health, A WHO contribution to the International Drinking Water Supply and Sanitation Decade, Geneva, WHO, 56 p.

WORLD HEALTH ORGANIZATION (1976): Surveillance of Drinking Water Quality, Monograph series no. 63, Geneva, WHO (specimen copy), 135 p.

- TRANSPORTS :

HAZARD, John L. (1977): Transportation: Management, Economics, Policy, Cambridge, Maryland, Cornwell Maritime Press Inc., $597 \mathrm{p}$.

MORICE, Lucien (1968): Les Transports fluviaux, Que sais-je ? n ${ }^{\circ}$ 540, Paris, PUF, 128 p.

SECRÉTARIAT D'ÉTAT AUX AFFAIRES ÉTRANGÈRES (1970): Les Différents Modes de transport, Paris, $238 \mathrm{p}$.

\section{ZONES TROPICALES / ZONES HUMIDES :}

BURGIS, M.J., BYMOENS, J.J. (1987): African Wetlands and Shallow Water Bodies/Zones humides et lacs peu profonds d'Afrique, Directory/Répertoire, Paris, Orstom, $650 \mathrm{p}$.

DUBROCA, E. (1985): Bibliographie des zones humides de France, Fasc. 26 Inven. fa. et fl., Paris, Secrétariat de la faune et de la flore, $138 \mathrm{p}$.

HADLEY, M., SCHRECKENBERG, K. (1989): Contributing to Sustained Resource Use in the Humid and Sub-humid Tropics. Some Research Approaches and Insights, Paris, Unesco, Mab digest 3, 59 p.

RIOU, G. (1990): L'Eau et les Sols dans les géosystèmes tropicaux - systèmes d'érosion hydrique, Coll. Géographie, Paris, Masson, $222 \mathrm{p}$.

\section{- DICTIONNAIRES / GLOSSAIRES :}

ABERCROMBIE, M., HICKMAN, C.J., JOHNSON, M.L. (1964): A Dictionary of Biology, United States, Aldine Publishing, 5th ed. revised, $254 \mathrm{p}$.

BUREAU DES TRADUCTIONS, DIRECTION GÉNÉRALE DE LA TERMINOLOGIE ET DE LA DOCUMENTATION (1977): Bulletin de terminologie, bateaux et engins de pêche/Boats and Fishing Gear, Bull. 158, Ottawa, Ministre des approvisionnements et services Canada, $521 \mathrm{p}$.

CARPENTER, R. (1938): An Ecological Glossary, New York, Hafner Publishing Company, 306 p.

CHALLINOR, J. (1964): A Dictionary of Geology, Cardiff, Great Britain, University of Wales Press, 2nd ed., $289 \mathrm{p}$.

COLLECTIF (1969): Encyclopédie internationale des sciences et des techniques en 10 volumes, France, Presses de la Cité, index.

COLLECTIF (1974): The World Book Encyclopedia, 21 Volumes, Chicago, Field Enterprises Educational Corporation.

CONSEIL INTERNATIONAL DE LA LANGUE FRANÇAISE (1989): Dictionnaire de l'océan, Paris, CILF, 758 p., index anglais-français, allemand-français.

CONSEIL INTERNATIONAL DE LA LANGUE FRANÇAISE (1979): Vocabulaire de la géomorphologie, Paris, Hachette, 218 p., index allemand et français. 
CONSEIL INTERNATIONAL DE LA LANGUE FRANÇAISE (1976): Vocabulaire de l'océanographie, Agence de coopération culturelle et technique, avec le concours du Conseil international de la langue française (CILF), Paris, Hachette, 431 p.

DAJET, Philippe, GODRON, Michel (1979) : Vocabulaire d'écologie, Paris, Hachette, $2^{2}$ éd. revue et complétée, $300 \mathrm{p}$.

DUVAL, C., DUVAL, C. (1978): Dictionnaire de la chimie et de ses applications, Paris, Technique et documentation, $3^{\mathrm{e}}$ éd., $1087 \mathrm{p}$.

FAIRBRIDGE, R.W. (1967): The Encyclopedia of Atmospheric Sciences and Astrogeology, New york, Reinhold Publishing Company.

FAIRBRIDGE, R.W. (1972): The Encyclopedia of Geochemistry and Environmental Sciences, Encyclopedia of Earth Sciences Series, Vol. IVA, London, Van Nostrand Reinhold Company, 1321 p.

FAIRBRIDGE, R.W. (1948): The Encyclopedia of Geomorphology, New York, Reinhold Book Company, $1295 \mathrm{p}$.

FAIRBRIDGE, R.W., FINKL, C.W. Jr. (1979): Encyclopedia of Soil Science Part I, Pennsylvania, USA, Dowden, Hutchinson \& Ross Inc., $646 \mathrm{p}$.

FAO (1981): Fish Culture / Pisciculture / Piscicultura, Terminology Bulletin 19, Rome, FAO, 151 p.

FOUCAULT, A., RAOULT, J.F. (1984): Dictionnaire de géologie, Paris, Masson, 347 p.

FOUCAULT, A., RAOULT, J.F. (1988): Dictionnaire de géologie, Paris, Masson, $3^{\mathrm{c}}$ é., 352 p.

GEORGE, P. (1970) : Dictionnaire de la géographie, Paris, PUF, 485 p.

GEORGE, P. (1984) : Dictionnaire de la géographie, Paris, PUF, $3^{e}$ éd. revue et augmentée, 485 p.

GRANDCHAMP-TUPULA, Mariette (1988): Lexique des barrages - Glossary on Dams, Bulletin de terminologie $\mathrm{n}^{\circ} 178$ / Terminology Bulletin 178, Ottawa, Approvisionnements et services Canada, $83 \mathrm{p}$.

GRANT, C., GRANT, R. (1987): Grant \& Hackle's Dictionary, London, McGraw-Hill, 5th ed., 641 p.

GRAY, P. (1970): The Encyclopedia of the Biological Sciences, London, Van Nostrand Reinhold, 1025 p.

GRUND, La Grande Encyclopédie du monde animal, Paris, 399 p.

HAMPEL, G.A., HAWLEY, G.G. (1982): Glossary of Chemical Terms, London, Van Nostrand Reinhold Company, 2nd ed., $306 \mathrm{p}$.

HAWLEY, G.G. (1981): The Condensed Chemical Dictionary, London (10th), 1135 p.

KENNETH, J.H. (1963): Henderson's Dictionary of Biological Terms, London, Oliver \& Boyd, 640 p.

LE PRAT, Guy (Éd.) (1981): Dictionnaire usuel de l'environnement et de l'écologie, t. I et II, Bibliothèque de l'environnement, Paris, Guy Le Prat, 135 p. +150 p.

McGRAW-HILL (1987): Encyclopedia of Science and Technology, 20 volumes, Paris, McGraw-Hill, 6th ed.

METRO, A. (1975): Dictionnaire forestier multilingue, Coll. Terminologie forestière multilingue $\mathrm{n}^{\circ} 2$, Paris, CILF, $433 \mathrm{p}$.

MICHEL, J.P., FAIRBRIDGE, R.W. (1980): Dictionnaire des sciences de la terre anglais-français/françaisanglais, USA, Paris, Masson Publishing Inc., $411 \mathrm{p}$.

OCDE (1978): Dictionnaire multilingue des poissons et de la pêche, Farnham, Surrey (G.B.), Fishing News Books Limited, 2nd ed., 430 p.

PARKER, S.P. (1989): McGraw-Hill Dictionary of Scientific and Technical Terms, New York, McGraw-Hill, 4th ed., $2088 \mathrm{p}$.

PARLEMENT EUROPÉEN (1977): Terminologie du secteur de la pêche, Direction de la traduction et de la terminologie, Luxembourg, 89 p., avec annexe.

PLAISANCE, G., CAILLEUX, A. (1958): Dictionnaire des sols, Paris, La maison rustique, Librairie agricole, horticole, forestière et ménagère, $605 \mathrm{p}$.

RICE, C.M. (1955): Dictionary of Geological Terms (Exclusive of Stratigraphic Formations and Paleontologic Genera and Species), USA, Michigan, Ann Arbor, Edwards Brothers, $461 \mathrm{p}$.

TOUFFET, J. (1982) : Dictionnaire d'écologie, Rennes, Ouest-France, 108 p.

\section{- ARTICLES DE PÉRIODIQUES :}

BRAVARD, J.P. (1985): «Le méandre de la mallourdie sur le Rhône, court-circuits de Chantagne (Savoie). Dynamique fluviale appliquée à l'écologie», Bull. Rhôd. de géomorphologie, 17, 18, Lyon, pp. 3-16.

BRAVARD, J.P., AMOROS, C., PAUTOU, G. (1986) "Impact of Civil Engineering Works on the Successions of Communities in a Fluvial System," Orkas, 47, pp. 92-111.

DEMANGEOT, J. (1945) : «Éléments de dynamique fluviale», Annal. Géographie, Paris, pp. 52-54.

EUROPEAN NETWORK OF SCIENTIFIC AND TECHNICAL COOPERATION (1988): "Fish and their Environment in Large European River Ecosystems," CCI Eau, 7 (1), pp. 1-154.

HARDIN, Garett (1960): “The Competitive Exclusion Principle," Science, 131, pp. 1292-1297.

KEMPF, C. (1974): «Aménagement des rivières et protection de la nature», Bulletin de la Société industrielle de Mulhouse, L'Eau en Alsace, $\mathrm{n}^{\circ} 4$, pp. 163-177. 
SCHUM, S.A. (1981): "Evolution and Response of the Fluvial System. Sedimentologic Implications," SEPM, Special Publication, $\mathrm{n}^{\circ} 31$, pp. 19-29.

SCHUM, S.A. (1985): "Patterns of Alluvial Rivers," Ann. Rev. Earth. Planel. Sci., 13, Washington, pp. 5-27. SCHUM, S.A. (1987): "River Responses," The Geol. of North America, Vol. K-3, chap. 10, pp. 221-240, North America and Adjacent Oceans during the Last Deglaciation, The Geol. Soc. of America.

SIEBUITH et al. (1978): "Pelagic Ecosystem Structure: Heterotrophic Compartments of the Plankton and their

Relationship to the Plankton Franctions," Limnological Oceanography, vol. 23 (6), The American Society of Limnology and Oceanography, pp. 1256-1263.

TANSLEY, A.G.: "The Use and Abuse of Vegetational Concepts and Terms," Ecol., 16, pp.284-307.

UNESCO (1988): "River: Lake and Shore," Man belongs to the earth, MAB, Unesco, Paris, pp. 128-140.

Philippe Thorron et André Clas 